https://helda.helsinki.fi

\title{
Imatinib mesylate does not counteract ovarian tissue fibrosis in postnatal rat ovary
}

\section{Asadi-Azarbaijani, Babak}

2019-06

Asadi-Azarbaijani , B , Braber , S, van Duursen , M , Jahnukainen , K, Santos , R \& Oskam , I C 2019 , ' Imatinib mesylate does not counteract ovarian tissue fibrosis in postnatal rat ovary ' , Reproductive Biology , vol. 19 , no. 2 , pp. 133-138 . https://doi.org/10.1016/j.repbio.2019.03.003

http://hdl.handle.net/10138/312985

https://doi.org/10.1016/j.repbio.2019.03.003

draft

Downloaded from Helda, University of Helsinki institutional repository.

This is an electronic reprint of the original article.

This reprint may differ from the original in pagination and typographic detail.

Please cite the original version. 
Original article

\title{
Imatinib mesylate does not counteract ovarian tissue fibrosis in postnatal rat ovary
}

\author{
Babak Asadi-Azarbaijani $^{\mathrm{a}, *}$, Saskia Braber ${ }^{\mathrm{b}}$, Majorie van Duursen ${ }^{\mathrm{c}}$, Kirsi Jahnukainen ${ }^{\mathrm{d}, \mathrm{e}}$, \\ Regiane Santos ${ }^{\mathrm{f}, \mathrm{g}}$, Irma Oskam ${ }^{\mathrm{h}}$ \\ ${ }^{a}$ VID Specialized University, Faculty of Health studies, Oslo, Norway \\ ${ }^{\mathrm{b}}$ Faculty of Veterinary Medicine, IRAS, Utrecht University, Utrecht, the Netherlands \\ ${ }^{\mathrm{c}}$ Department of Environment and Health, Faculty of Science, Vrije Universiteit Amsterdam, Amsterdam, the Netherlands \\ ${ }^{\mathrm{d}}$ New Children's Hospital, Pediatric Research Center, University of Helsinki and Helsinki University Hospital, Helsinki, Finland \\ ${ }^{\mathrm{e}}$ Department of Women's and Children's Health, NORDFERTIL Research Lab, Stockholm, Sweden \\ ${ }^{\mathrm{f}}$ Laboratory of Wild Animal Biology and Medicine, Federal University of Pará, Castanhal, Pará, Brazil \\ ${ }^{\mathrm{g}}$ Schothorst Feed Research, Lelystad, the Netherlands \\ ${ }^{\mathrm{h}}$ The production animal experimental research centre (SHF), Norwegian University of Life Sciences, Oslo, Norway
}

\section{A R T I C L E I N F O}

\section{Keywords:}

Imatinib

Tissue fibrosis

Ovary

FOXO1

FOXO3

\begin{abstract}
A B S T R A C T
Chemotherapy may result in ovarian atrophy, a depletion of the primordial follicle pool, diminished ovarian weight, cortical and stromal fibrosis. Imatinib mesylate is an anticancer agent that inhibits competitively several receptor tyrosine kinases (RTKs). RTKs play important roles in cell metabolism, proliferation, and apoptosis. In clinic, imatinib mesylate is also known as an anti-fibrotic medicine. In the present study, the impact of imatinib on the ovarian tissue was investigated by assessing ovarian tissue fibrosis in postnatal rat administered with or without imatinib for three days. Fibrosis in the ovarian tissue was determined by histology (Picrosirius and Masson's trichrome staining) and the protein expression of vimentin and alpha-smooth muscle actin ( $\alpha$-SMA). Furthermore, mRNA expression of Forkhead box transcription factor O1 and O3 (FOXO1 and FOXO3), which are markers of cell proliferation was quantified. A short-term exposure to imatinib showed to increase tissue fibrosis in ovaries. This was observed by Masson's trichrome staining. Exposure to imatinib led also to a down-regulation of vimentin protein expression and up-regulation mRNA expression of FOXO3. This may indicate a role of FOXO3 in ovarian tissue fibrosis in postnatal rat ovaries.
\end{abstract}

\section{Introduction}

The ovarian cortex is composed by connective tissue cells and collagen fibers, which are surrounding the ovarian follicles of different developmental stages. The medulla is described as highly vascularized stroma usually in the center of the ovary, consisting of connective tissue, muscle cells, elastic and reticular fibers [1,2]. Development and maturation of ovarian follicles are related to a communication between cortex and medulla. Ovarian injury caused by different factors, such as surgery, chemotherapy, radiotherapy, inflammation, and immune abnormalities can trigger ovarian fibrosis [3-5]. Ovarian tissue fibrosis can lead to functional decline of the ovary and even premature ovarian failure [3,5].

Fibrosis is the formation of excess fibrous connective tissue in an organ or tissue, which attributes to the excessive deposition of extracellular matrix components [6,7]. Different factors have been identified as important regulators of fibrosis such as; cytokines (IL-13, IL-21, TGF$\beta 1$ ), chemokines (MCP-1, MIP-1 $\beta$ ), angiogenic factors (VEGF), growth factors (PDGF), peroxisome proliferator-activated receptors (PPARs), acute phase proteins (SAP), caspases, components of the renin-angiotensin-aldosterone system (ANG II), and Forkhead box $\mathrm{O}$ transcription factors (FOXOs) [5,8-11].

It is shown that some anticancer drugs are able to counteract the cancer cells without causing fibrosis [12,13]. Imatinib mesylate (STI517, Glivec ${ }^{\circledast}$ ), a tyrosine kinase inhibitor, is known as a targeted anticancer agent to treat several cancers by blocking receptor tyrosine kinases to inhibit cell proliferation [14,15]. In clinic, imatinib mesylate is also used as an anti-fibrotic medicine, for example during treatment of idiopathic pulmonary fibrosis [13].

The effect of chemotherapeutic agents on ovarian function should include evaluation of the whole ovary including ovarian follicles, cortical tissue and stromal tissue. In our previous study, we have reported

\footnotetext{
* Corresponding author at: VID Specialized University, Faculty of Health studies, PB 184, 0319 Vinderen, Oslo, Norway.

E-mail address: babak.asadi@hotmail.com (B. Asadi-Azarbaijani).
} 
that imatinib negatively affects early folliculogenesis in postnatal rat ovaries [16]. However, effects of imatinib on ovarian fibrosis on postnatal rats are not known.

In the current study effect of the tyrosine kinase inhibitor, imatinib on ovarian fibrosis were evaluated after short-term in vivo exposure of healthy postnatal rats.

\section{Materials and methods}

\subsection{Animals and study design}

All experiments were performed on postnatal Sprague Dawley female rats. The animals $(n=28)$ were born and maintained under the standard life conditions for 5 days at the Animal Center of the Norwegian Institute of Public Health, Oslo, Norway. Ethical permission for drug treatment was obtained from the Norwegian Institute of Public Health, Oslo, Norway. From day 2 to 4 , the treatment groups $(n=20)$ were treated daily by imatinib mesylate (Glivec ${ }^{\circledR}$, STI571, Novartis Pharmaceuticals Corporation, Switzerland) $(150 \mathrm{mg} / \mathrm{kg})$ dissolved in water with respect to the rats' weight $(1.2-2.1 \mathrm{mg})$. Both treated and control animals $(n=8)$ were euthanized on day 5 . Bilateral ovariectomy was performed and ovarian samples were collected for histological, immunofluorescence and mRNA expression analysis at the Faculty of Veterinary Medicine, Utrecht University, Utrecht, The Netherlands. The full procedure is described in previous studies $[16,17]$.

\subsection{Tissue fibrosis}

\subsubsection{Picrosirius red staining}

To investigate the structure of ovarian tissue, relative areas of fibrosis were evaluated by Picrosirius red staining plus polarization microscopy in Bouins' fixed samples [18]. For this, ovaries from 28 rats (eight from the control group and 20 from the imatinib treated group) were stained. Picrosirius red is a strong anionic dye that attaches to collagen fibers, thus fibrotic areas become easily recognizable [18]. When evaluated by light microscopy, collagen is stained red, while cytoplasm is stained in yellow. Under polarized light microscopy, collagen type I is stained yellow orange birefringence, while collagen type III is stained green birefringence. In brief, collagen types I and III, were calculated as a percentage of the area of each image (expressed in pixels). To avoid counting non-collagen fibers, a color separation on the original (circularly polarized) images, resolving each into its cyan, yellow, magenta and black components. Fibrotic area calculation was performed automatically with the help of the image processing and analysis software Image $J$ (National Institutes of Health, USA).

\subsubsection{Masson's trichrome staining}

Masson's Trichrome stain was performed to determine fibrotic areas in the ovarian tissue by identifying the relative levels of newly formed collagen $[18,19]$. Ovaries (six from each group, i.c. control and imatinib treated) were fixed in Bouins' solution and embedded in paraffin. The $4 \mu \mathrm{m}$ deparaffinized sections were first stained with an acid dye (acid fuchsin), treated with phosphomolybdic acid and finally stained with methyl blue. Old collagen was stained in red, while newly formed collagen fibers were stained in blue. Newly formed collagen fibers were stained in blue. Photomicrographs from the Masson's trichrome-stained sections were taken with an Olympus BX50 microscope equipped with a Leica DFC 320 digital camera (magnification of 100x). As for Picrosirius red staining, fibrotic area calculation was performed automatically with the help of the image processing and analysis software Image $J$ (National Institutes of Health, USA).

\subsection{Immunohistochemical and immunofluorescence labelling}

The presence of fibrosis in ovarian tissue can be investigated by the determination of protein expression of the structural protein vimentin, as well as alpha-smooth muscle actin ( $\alpha$-SMA) as mesenchymal and myofibroblast markers [18,20,21]. Ovaries (six from each group, i.c. control and imatinib treated) fixed in $10 \%$ neutral buffered formalin were embedded in paraffin, sectioned in $4 \mu \mathrm{m}$ and deparaffinized. The endogenous peroxidase activity was blocked with $0.3 \% \mathrm{H}_{2} \mathrm{O}_{2}$ (Merck) in methanol for $30 \mathrm{~min}$ at room temperature and rehydrated in a graded ethanol series to Phosphate Buffer Saline (PBS). For antigen retrieval, the slides were boiled for $10 \mathrm{~min}$ in $10 \mathrm{mM}$ citrate buffer ( $\mathrm{pH} \mathrm{6.0)}$ ) in a microwave. The slides were cooled down to room temperature, rinsed with PBS and blocked with $5 \%$ goat serum for $30 \mathrm{~min}$ at room temperature. The tissue sections were incubated overnight $\left(4^{\circ} \mathrm{C}\right)$ with the primary antibodies: $\alpha$-smooth muscle actin ( $\alpha$-SMA, Abcam AB5694, 1:100) or vimentin (Abcam AB24525, 1:200) diluted in PBS containing $1 \%$ Bovine Serum Albumin. The negative controls lacking the primary antibodies were included. Sections were rinsed with PBS followed by incubation with Alexa-Fluor conjugated secondary antibodies (1:200, Invitrogen). After washing, the sections were mounted with Prolong Gold antifade reagent with 4',6-diamidino-2-phenylindole (DAPI) (Thermo Fisher Scientific), immunolocalization was visualized and images were acquired using the Keyence BZ-9000 microscope (magnification of 200x). Labelled area was calculated with the help of the image processing and analysis software Image $J$ (National Institutes of Health, USA). The relative expression of these proteins was calculated based on the fold difference in their expression in imatinib treated ovaries relative to the control ones.

\subsection{Quantitative real time polymerase chain reaction ( $q R T-P C R)$}

Total RNA from postnatal rat ovaries (six controls and 11 from imatinib treated) was extracted by using the Trizol reagent (Invitrogen, Carlsbad, CA) followed by PureLink ${ }^{\mathrm{TM}}$ RNA Mini Kit (Anbion ${ }^{\circledast}$ ), according to the manufacturer's instructions. The RNA concentration was estimated by reading the absorbance at $260 \mathrm{~nm}$ and was checked for purity at $280 \mathrm{~nm}$ in a spectrophotometer (NanoDrop ${ }^{\circledR} 2000$, Thermo Fisher Scientific Inc., Waltham, MA, USA). For each sample, the RNA concentrations were adjusted to $30 \mathrm{ng} / \mathrm{ml}$ and were used to synthesize cDNA. The reverse transcription was performed with the SuperScript III RT-PCR kit (Invitrogen). The mixture was incubated at $42{ }^{\circ} \mathrm{C}$ for $1 \mathrm{~h}$, subsequently at $80^{\circ} \mathrm{C}$ for $5 \mathrm{~min}$, and finally stored at $-20^{\circ} \mathrm{C}$. The negative control was prepared under the same conditions, but without addition of the nucleic acid. qRT-PCR was performed using a thermocycler (iQ5, Bio-Rad, Hercules, CA, USA). Primers (Table 1) derived from the Rattus norvegicus gene were designed using the Pick Primers designing tool from the National Center for Biotechnology Information GenBank (NCBI) or based on literature (reference genes; Table 1). A NCBI basic local alignment search tool (BLAST) ensured the specificity of primer sequences. The primers were tested according to the efficiency by qPCR analysis of a dilution series of pooled cDNA at a temperature gradient $\left(55-65^{\circ} \mathrm{C}\right)$ for primer annealing and subsequent melting curve analysis. The reaction mixture for the qPCR contained $0.5 \mathrm{mg}$ cDNA in sterile water $(7.4 \mathrm{ml}), 10 \mathrm{ml}$ iQSYBR Green Supermix (Bio Rad Laboratories Inc.), forward $(0.8 \mathrm{ml})$ and reverse $(0.8 \mathrm{ml})$ primers (final concentration of $0.4 \mathrm{pmol} / \mathrm{ml}$ for each primer), according to the manufacturer's instructions. Data were analyzed using the efficiency corrected Delta-Delta-Ct method (Pfaffl MW, 2001). The fold-change values of the target genes were normalized using the geometric average of the fold-change values of two reference genes: HPRT and SDHA (Table 1). The reactions for PCR amplification consisted of initial denaturation and polymerase activation for $15 \mathrm{~min}$ at $94^{\circ} \mathrm{C}$, followed by 40 cycles of $15 \mathrm{~s}$ at $94^{\circ} \mathrm{C}, 30 \mathrm{~s}$ at $50{ }^{\circ} \mathrm{C}, 45 \mathrm{~s}$ at $72{ }^{\circ} \mathrm{C}, 15 \mathrm{~s}$ at $95^{\circ} \mathrm{C}$ and $1 \mathrm{~min}$ at $60^{\circ} \mathrm{C}$. The method qRT-PCR was performed by using the thermal cycler CFX96 real-time PCR detection system (Bio-Rad) and CFX Manager System Software Version 3.0 (Bio Rad). FOXO1 and FOXO3 were used as the markers for cell proliferation [22,23]. The fold-change values of the genes encoding FOXO1 and FOXO3 were 
Table 1

Primers used for the mRNA quantification of reference genes (stably expressed) and genes of interest.

\begin{tabular}{|c|c|c|c|c|}
\hline Genes & Accession $n^{\circ}$ & Primer & Product size $(\mathrm{pb})$ & Source \\
\hline \multicolumn{5}{|c|}{ Reference } \\
\hline HPRT & NM_012583.2 & $\begin{array}{l}\text { F - GCTGAAGATTTGGAAAAGGTG } \\
\text { R - AATCCAGCAGGTCAGCAAAG }\end{array}$ & 157 & Hvid et al., 2011 \\
\hline SDHA & NM_130428.1 & $\begin{array}{l}\text { F - AGACGTTTGACAGGGGAATG } \\
\text { R - TCATCAATCCGCACCTTGTA }\end{array}$ & 160 & Hvid et al., 2011 \\
\hline \multicolumn{5}{|l|}{ Target } \\
\hline FOXO1 & NM_001191846.2 & $\begin{array}{l}\text { F - TGGGTGTCAGGCTAGGAGTT } \\
\text { R - GGCATCTTTGGACTGCTCCT }\end{array}$ & 92 & This manuscript \\
\hline FOXO3 & NM_001106395.1 & $\begin{array}{l}\text { F - AACAGTACCGTGTTCGGACC } \\
\text { R - AGTGTCTGGTTGCCGTAGTG }\end{array}$ & 119 & This manuscript \\
\hline
\end{tabular}

normalized using the geometric average of the fold-change values of the two reference genes: HPRT and SDHA (Table 1). The relative expression of FOXO1 and FOXO3 was calculated based on the fold difference in their expression in imatinib treated ovaries relative to the control ones.

\subsection{Statistical analysis}

In all cases, the statistical tests were performed by using software GraphPad Prism 6. Relative areas of ovarian tissue fibrosis, alpha-SMA, vimentin, as well as mRNA expression were compared between control and treated groups with the non-parametric Mann-Whitney test. All data are presented as mean values \pm SEM, the standard errors being calculated from the variance between samples. P-values $\leq 0.05$ indicated statistical significance.

\section{Results}

\subsection{Picrosirius and Masson's trichrome staining}

Although Picrosirius red staining showed that the total tissue fibrosis increased from $20.1 \pm 5.8 \%$ in the control group to $39.2 \pm 4.1 \%$ in the imatinib treated group, this difference was not significant $(\mathrm{P}>0.05)$. Similarly, the levels of collagen I increased from $0.26 \pm 0.15 \%$ in the control group to $0.48 \pm 0.12 \%$ in the imatinib treated group, while the levels of collagen III increased from $0.23 \pm 0.14 \%$ in the control group to $0.47 \pm 0.13 \%$ in the imatinib treated group, but none of these differences were significant ( $\mathrm{P}>0.05$ ) (Fig. 1). However, when the ratio of newly formed collagen: total evaluated tissue section was determined based on Masson's trichrome staining, a significant $(P<0.0152)$ increase in newly formed fibers was observed, where it increased from $0.29 \pm 0.11 \%$ in the control group to $1.67 \pm 0.66 \%$ in the imatinib treated group (Fig. 1).

\subsection{Vimentin and $\alpha$-SMA protein expression}

Imatinib treatment did not affect $\alpha$-SMA protein expression. However, a significant $(\mathrm{P}<0.041)$ decrease $(2.1$-fold $)$ was observed when vimentin expression was determined in imatinib treated ovarian tissues when compared with control ones (Fig. 2).

\section{3. mRNA expression of FOXO1 and FOXO3}

The relative mRNA expression levels of FOXO1 and FOXO3 genes are depicted in Fig. 3. FOXO1 mRNA expression was not affected by imatinib treatment. However, mRNA expression of FOXO3 increased ( $>$ 5-fold) in imatinib treated ovaries when compared to control.

\section{Discussion}

Ovarian fibrosis is characterized by excessive proliferation of ovarian fibroblasts and in addition with the deposition of extracellular matrix, being one of the main reasons for ovarian dysfunction [3,5,24]. A complex network is involved in the development and progression of tissue fibrosis. The exact mechanisms involved in ovarian damage by anticancer treatment are still unclear. However, it is known that ovarian damage includes follicular apoptosis as well as cortical fibrosis $[3,24]$.

Imatinib mesylate is known as an anticancer and anti-fibrotic medicine to treat several cancers by blocking the activity of several RTKs [13-15]. It is suggested that the effect of imatinib may be based on its role as an Abelson tyrosine kinase (c-Abl) inhibitor via PI3K/ PTEN/Akt signaling pathways $[14,15,25]$. There is a controversy in literature about the effect of imatinib on ovarian function. The initial reports have shown the protective effect of this drug on chemotherapyinduced follicle loss in mouse ovary, whereas several other studies have documented inconsistent results [25-30]. Recently, Bildik et al, showed molecular evidence for toxicity of imatinib in human ovary [31].

However, anti-fibrotic effect of imatinib on ovarian fibrosis is still unknown. In the present study, three days imatinib treatment during early folliculogenesis increased tissue fibrosis in postnatal rat ovaries. This was concomitantly with up-regulation mRNA expression of FOXO3 together with a down-regulation of vimentin protein expression.

Fibrosis can obliterate the architecture and function of the underlying organ or tissue, and it is attributed to an excess in the deposition of extracellular matrix components, including collagen, in the tissue [6]. Besides the fact that collagen supports cell structure and architecture in tissues, it also may act as a signaling molecule, activating specific receptors at the cell surface [32].

Among the different methods to assess tissue fibrosis in ovarian tissue, Picrosirius red staining and Masson's trichome are the most used [33-35]. Picrosirius red is an anionic dye that can associate with collagen fibers, which are cationic. Picrosirius red is able to bind the total fibers giving a different birefringence to collagen types I and III in tissues with advanced stages of fibrosis [36]. On the other hand, Masson's trichome staining is used to detect newly formed collagen $[19,33]$. In a previous study with ovarian autografts, it was possible to observe that the rate of tissue fibrosis detected by Pricosirius Red staining was much higher than that determined with Masson's trichome [35]. Furthermore, Gavish et al. used also Masson's trichome staining to detect early fibrosis in ovarian grafts [33]. These studies confirm that Masson's trichome is more indicated for the assessment of recently formed collagen, as observed in our study. However, none of these methods could show significant changes in collagen I and III in postnatal rat ovaries after exposure to imatinib.

Studies have identified collagen as an endogenous ligand for the discoid in domain receptors 1 and 2 (DDR1 and DDR2) [32,37]. Both DDR1 and DDR2 are tyrosine kinase receptors [38]. Briefly, collagen binding to DDR1 induces tyrosine kinase phosphorylation and may participate in mammary gland formation and breast cancer, whereas DDR2 is activated only by fibrillary collagens, which act as its endogenous ligand $[32,37,38]$. Matsumura et al. have indicated DDR2 as 

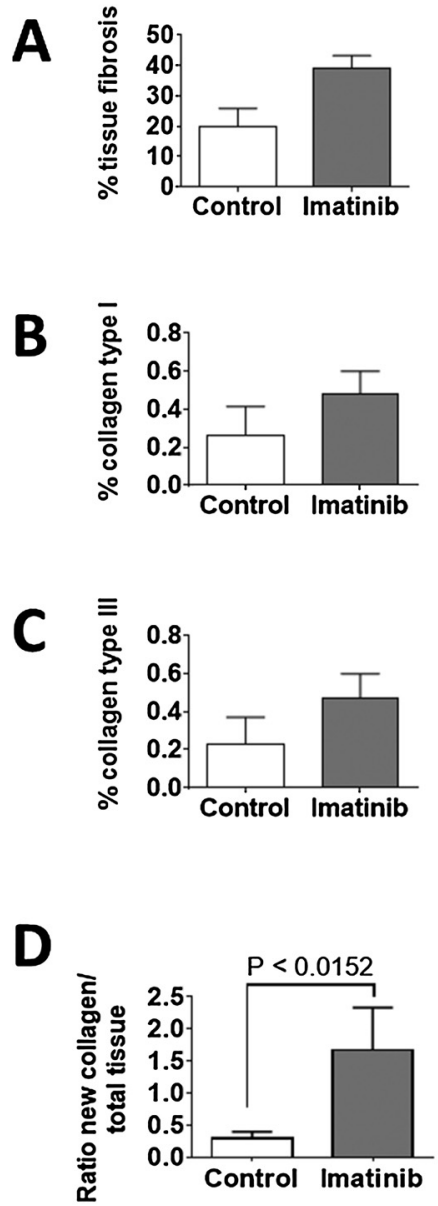
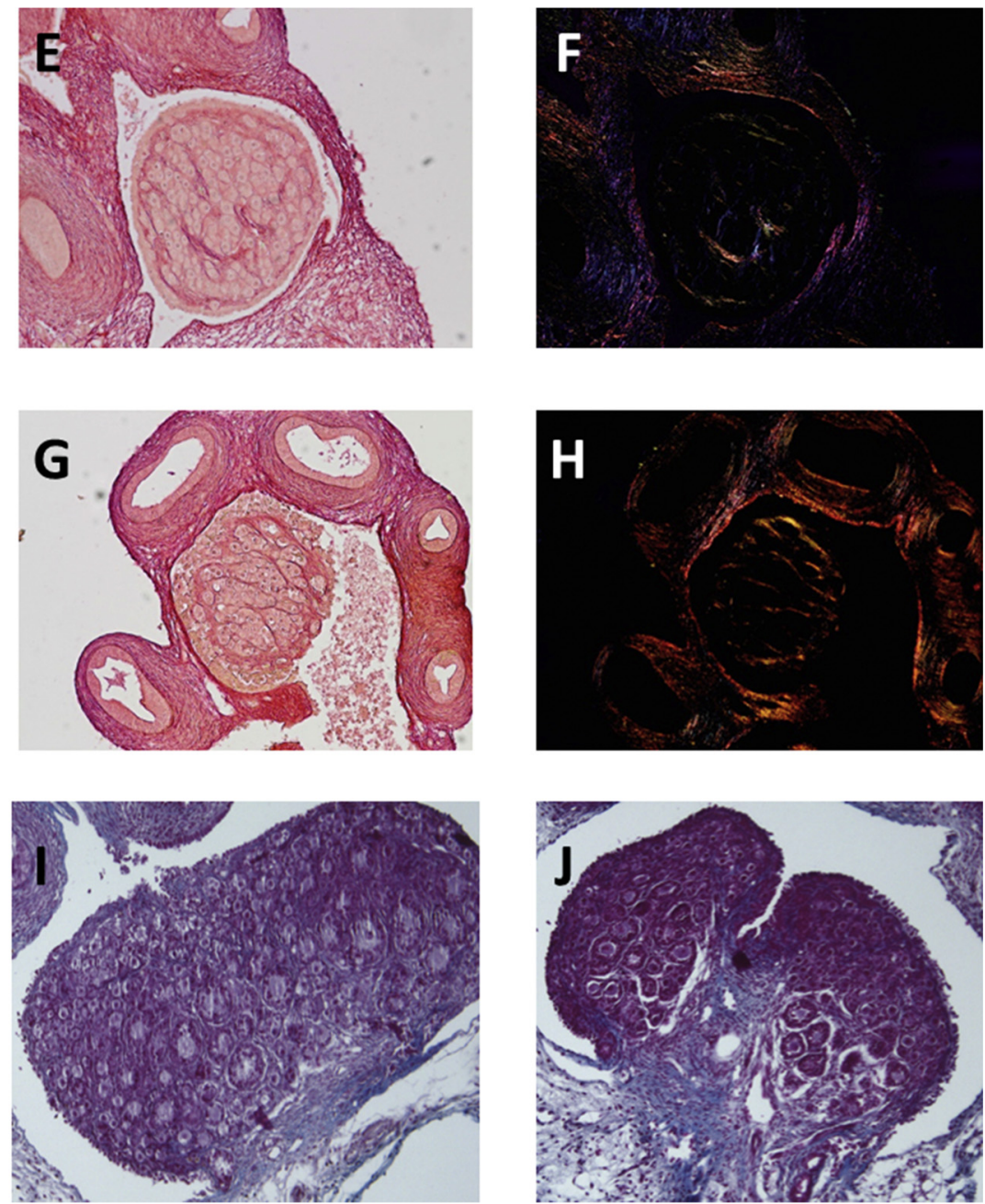

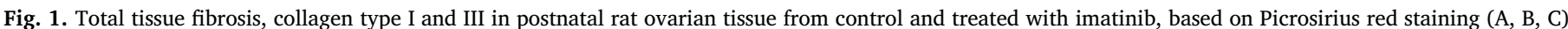

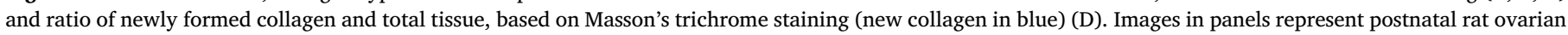
tissue from control (E, F, I) and treated imewith imatinib (G, H, J).
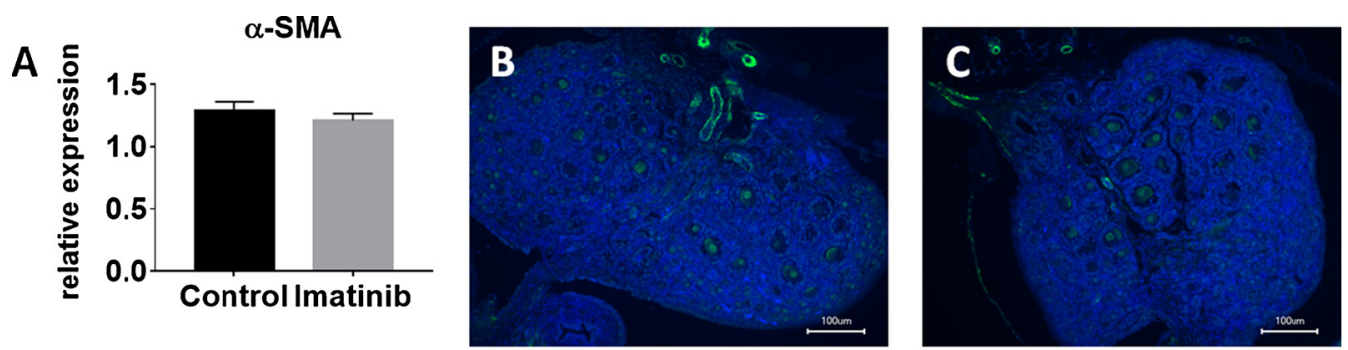

D
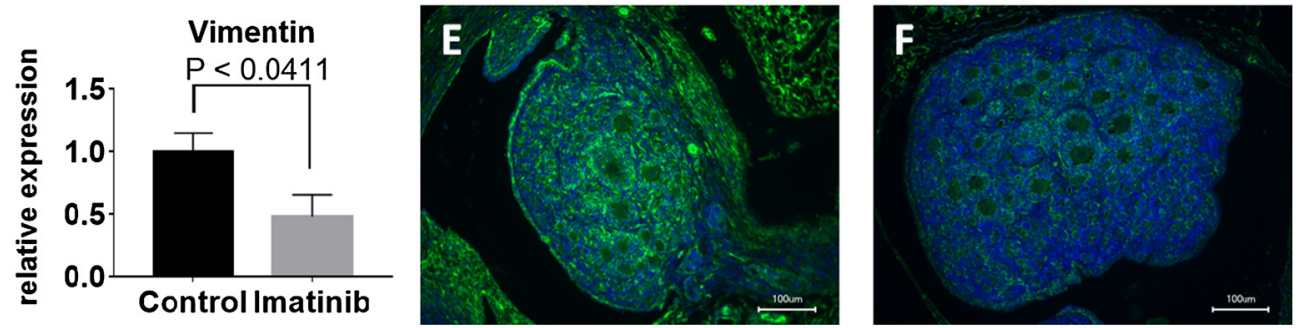

Fig. 2. Immunolabelling of $\alpha$-SMA (A-C) and vimentin (D-F) proteins in ovarian tissue from control group (B, E) and treated with imatinib (C, F). The relaive expression of these proteins is expressed as fold-change in levels of $\alpha$-SMA (A) and vimentin (D) in postnatal rat ovaries from control and imatinib treated animals. 

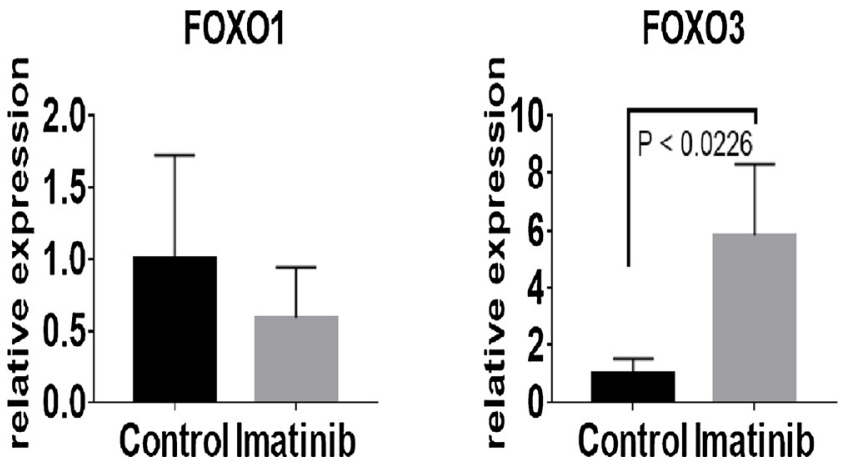

Fig. 3. The fold-change in mRNA expression levels of FOXO1 (A) and FOXO3 (B) genes in postnatal rat ovaries from control and imatinib treated animals.

a critical player in ovarian function and regulation, such as anti-apoptosis, steroid synthesis, gonadal development, ovulatory cycles and ovulation [39]. Imatinib is known to inhibit DDRs and regress fibrosis in mature mammals with cancer $[40,41]$. In the present study, no significant differences of collagen types I and III between imatinib treated group and the control groups were observed in postnatal rat ovary during three days treatment (Fig. 1). However, differences were observed in the early tissue fibrosis. We may conjecture that the total amount of collagen may increase over time. Further studies with a longer treatment duration may show different results.

FOXO transcription factors are key regulators of cellular metabolism, proliferation, apoptosis and stress resistance [22]. In ovaries, inactivity or mutation of these transcription factors have been related to premature ovarian failure [42]. Transcription factors FOXO1 and FOXO3 in rodents are highly expressed respectively in granulosa cells and oocytes at different stages of follicle development [43]. They play a role, not solely on primordial follicle activation, but also on oocyte maturation, ovulation and luteinization $[43,44]$. Inactivity or mutation of these transcription factors has been related to POF [42]. The effect of imatinib is based on binding to c-Abl kinase and inactivating the PI3K/ Akt/FOXO axis $[28,45]$. FOXOs have also shown to play a potential role in tissue fibrosis in other organs e.g. heart, lungs and liver $[11,23,46,47]$. As far as we know, the role of FOXOs in ovarian tissue fibrosis has not been previously described.

In this study, FOXO1 was not affected, while FOXO3 was up-regulated in the imatinib treated postnatal rat ovaries. Activated Akt phosphorylates a variety of effector molecules including FOXO1, which then control cell survival and growth [48]. FOXO1 is mostly known as regulator of cell cycle progression and apoptosis via KL/c-Kit and PI3K signaling pathways [43]. FOXO1 is important for wound healing by decreasing fibroblast proliferation [49,50]. Akt phosphorylates and inactivates FOXO3a by promoting translocation to the cytoplasm, which promotes proliferation [48]. Studies on the role of FOXO3 on fibrosis suggest that inactivation of this factor is associated with a decreased fibrosis, probably by regulating proliferation and apoptosis sensitivity [51-53]. FOXO3a deficiency protects idiopathic Pulmonary Fibrosis fibroblasts from type I polymerized collagen matrix-induced apoptosis via caveolin-1 and Fas [11].

Imatinib inhibit FOXO3 phosphorylation, i.e. its translocation to cytoplasm of primordial follicles in vitro via PI3K/Akt pathway [45,54]. However, besides phosphorylation, FOXO3 can be also regulated by acetylation, methylation, glycosylation, redox modulation, and ubiquitination, which will determine FOXO3 function [55]. The higher expression of FOXO3 in imatinib treated postnatal rat ovaries may be related to ovarian tissue fibrosis in the current study. This is contradictory with the anti-fibrotic effect of imatinib on other type of tissues compared with ovarian tissue in postnatal rats $[51-53,56]$. Therefore, the precise role of $\mathrm{FOXO} 3$ in ovarian tissues remains unclear, and it could be reasonable to postulate that FOXO3 may have another role in ovarian tissue fibrosis in postnatal rats. In spite of high similarity among RTKs, it seems there are differences within their activation, which could be exploited to obtain kinase selective compounds. These differences may depend on species selection and/or some other factors such as age, type of tissue, dose and duration of treatments.

During fibrogenesis, mutually interacting factors disrupt the balance between synthesis and degradation of extracellular matrix [5]. In addition, over-proliferation of ovarian mesenchymal fibroblasts and the excessive deposition of extracellular matrix may be stimulated [5]. Imatinib inhibits the vascular endothelial growth factor (VEGF), which in its turn plays a role in fibrosis by upregulating the expression of collagen and $\alpha$-SMA $[5,57]$. Luo et al. found that $\alpha$-SMA directly regulates several profibrotic and immune cytokine genes in hepatic stellate cells [58]. Protein $\alpha$-SMA is associated with enhanced collagen secretion and, therefore, represents a critical transition in fibrogenesis [59]. (Challa and Stefanovic 2011, Hinz 2016) In the present study, imatinib did not affect the expression of $\alpha$-SMA. This shows that fibrosis may occur via a different pathway. On the other hand, vimentin expression was reduced significantly in imatinib treated postnatal rat ovaries. Vimentin filaments are important in the development of tissue fibrosis by stabilizing the collagen I and promoting fibroblasts migration [60,61]. Disruption of vimentin reduces synthesis of type I collagen [60]. From a therapeutic point of view, the increased collagen expression has a central role in the development of tissue fibrosis [60]. Vimentin filaments are known as a target for anti-fibrotic therapy where imatinib treatment prevents tissue fibrosis by reducing the vimentin expression $[60,62]$. As expected, the present study showed a decrease in vimentin expression caused by imatinib administration. However, without avoiding ovarian tissue fibrosis. Vimentin is involved in sequestering and degradation of FOXO3 [61]. Hence, this finding shows that fibrosis does not only follow a vimentin-dependent pathway in postnatal rat ovaries and other targets than collagen type I should be considered.

The present study suggests that anti-fibrotic effects of imatinib in ovaries may be age-dependent. Observation is in line with previous reports where imatinib has shown to interfere with physiological signal cascades and this modulation has varied significantly with age and maturational status $[17,63]$. However, further studies are needed to evaluate differences in mechanism of ovarian fibrosis at different maturational stages with a longer treatment duration. Consequently, before introducing imatinib as an anti-fibrotic drug we need more information about the complicated mechanism action of this drug in different tissues and its possible age-dependent adverse effects on ovarian stroma.

\section{Conclusion}

In conclusion, increased tissue fibrosis in postnatal rat ovaries treated with imatinib is probably related to a down-regulation of vimentin protein expression and up-regulation mRNA expression of FOXO3. More investigation of other related up/downstream proteins involved in fibrosis in postnatal rat ovaries might strengthen this observation.

\section{Conflicts of interest}

The authors declare no conflicts of interest.

\section{Sources of funding}

This research was supported by grants from the Norwegian National Advisory Unit on Women's Health, Oslo University Hospital, Norway and Faculty of Veterinary Medicine, IRAS, Utrecht University, Utrecht, the Netherlands. 


\section{Transparency document}

The Transparency document associated with this article can be found in the online version.

\section{Acknowledgment}

We would like to thank Trude Olsen at Norwegian Institute of Public Health, Oslo, Norway for her help at animal house.

\section{References}

[1] Bochner F, et al. A novel intravital imaging window for longitudinal microscopy of the mouse ovary. Sci Rep 2015;5:12446.

[2] Kuehnel W. Color atlas of cytology, histology, and microscopic anatomy. 4. edition, revised and enlarged. ed. thieme flexibook ix. Stuttgart/New York: Thieme; 2003. p. $534 s$

[3] Meirow D, et al. Cortical fibrosis and blood-vessels damage in human ovaries exposed to chemotherapy. Potential mechanisms of ovarian injury. Hum Reprod 2007;22(6):1626-33.

[4] Meirow D, et al. Searching for evidence of disease and malignant cell contamination in ovarian tissue stored from hematologic cancer patients. Hum Reprod 2008;23(5):1007-13.

[5] Zhou F, Shi LB, Zhang SY. Ovarian fibrosis: A phenomenon of concern. Chin Med J (Engl) 2017;130(3):365-71.

[6] Wynn TA. Cellular and molecular mechanisms of fibrosis. J Patho 2008;214(2):199-210

[7] Revelon G, et al. Focal fibrosis of the breast: imaging characteristics and histopathologic correlation. Radiology 2000;216(1):255-9.

[8] Wynn TA. Common and unique mechanisms regulate fibrosis in various fibroproliferative diseases. J Clin Invest 2007;117(3):524-9.

[9] Tomasek JJ, et al. Myofibroblasts and mechano-regulation of connective tissue re modelling. Nat Rev Mol Cell Biol 2002;3(5):349-63.

[10] Friedman SL. mechanisms of disease: mechanisms of hepatic fibrosis and therapeutic implications. Nat Clin Pract Gastroenterol Hepatol 2004;1(2):98-105.

[11] Nho RS, et al. Pathological alteration of FoxO3a activity promotes idiopathic pulmonary fibrosis fibroblast proliferation on type i collagen matrix. Am J Pathol 2011;179(5):2420-30.

[12] Torres VE, Leof EB. Fibrosis, regeneration, and aging: playing chess with evolution J Am Soc Nephrol 2011;22(8):1393-6.

[13] Beyer C, Distler JH. Tyrosine kinase signaling in fibrotic disorders: translation of basic research to human disease. Biochim Biophys Acta 2013;1832(7):897-904.

[14] Buchdunger E, et al. Abl protein-tyrosine kinase inhibitor STI571 inhibits in vitro signal transduction mediated by c-kit and platelet-derived growth factor receptors. J Pharmacol Exp Ther 2000;295(1):139-45.

[15] Deininger M, Buchdunger E, Druker BJ. The development of imatinib as a therapeutic agent for chronic myeloid leukemia. Blood 2005;105(7):2640-53.

[16] Asadi-Azarbaijani B, et al. Developmental effects of imatinib mesylate on follicle assembly and early activation of primordial follicle pool in postnatal rat ovary. Reprod Biol 2017;17(1):25-33.

[17] Nurmio M, et al. Receptor tyrosine kinase inhibition causes simultaneous bone loss and excess bone formation within growing bone in rats. Toxicol Appl Pharmacol 2011;254(3):267-79.

[18] Liu X, et al. Corroborating evidence for platelet-induced epithelial-mesenchymal transition and fibroblast-to-myofibroblast transdifferentiation in the development of adenomyosis. Hum Reprod 2016;31(4):734-49.

[19] Guo X, et al. Grafting of mesenchymal stem cell-seeded small intestinal submucosa to repair the deep partial-thickness burns. Connect Tissue Res 2016;57(5):388-97.

[20] Huang Y, et al. Enhanced efficacy and specificity of epithelial ovarian carcinogenesis by embedding a DMBA-coated cloth strip in the ovary of rat. J Ovarian Res 2012;5(1):21.

[21] Chen CM, Chang JL, Behringer RR. Tumor formation in p53 mutant ovaries transplanted into wild-type female hosts. Oncogene 2004;23(46):7722-5.

[22] Martins R, Lithgow GJ, Link W. Long live FOXO: unraveling the role of FOXO proteins in aging and longevity. Aging Cell 2016;15(2):196-207.

[23] Xin Z, et al. FOXO1/3: potential suppressors of fibrosis. Ageing Res Rev 2018;41:42-52.

[24] Meirow D, et al. Toxicity of chemotherapy and radiation on female reproduction. Clin Obstet Gynecol 2010;53(4):727-39.

[25] Gonfloni S, et al. Inhibition of the c-Abl-TAp63 pathway protects mouse oocytes from chemotherapy-induced death. Nat Med 2009;15(10):1179-85.

[26] Kerr JB, et al. Cisplatin-induced primordial follicle oocyte killing and loss of fertility are not prevented by imatinib. Nat Med 2012:18(8):1170-2.

[27] Carlsson IB, et al. Kit ligand and c-Kit are expressed during early human ovarian follicular development and their interaction is required for the survival of follicles in long-term culture. Reproduction 2006;131(4):641-9.

[28] Kim SY, et al. Rescue of platinum-damaged oocytes from programmed cell death through inactivation of the p53 family signaling network. Cell Death Differ 2013;20(8):987-97.

[29] Morgan S, et al. Cisplatin and doxorubicin induce distinct mechanisms of ovarian follicle loss; imatinib provides selective protection only against cisplatin. PLoS One 2013;8(7):e70117.

[30] Tuppi M, et al. Oocyte DNA damage quality control requires consecutive interplay of CHK2 and CK1 to activate p63. Nat Struct Mol Biol 2018;25(3):261-9.

[31] Bildik G, et al. C-Abl is not activated in DNA damage-induced and Tap63-mediated oocyte apoptosis in human ovary. Cell Death Dis 2018;9(10):943.

[32] Leitinger B, Hohenester E. Mammalian collagen receptors. Matrix Biol 2007;26(3):146-55

[33] Gavish Z, et al. Follicle activation and' burn-out' contribute to post-transplantation follicle loss in ovarian tissue grafts: the effect of graft thickness. Hum Reprod 2015;30(4):1003.

[34] Briley SM, et al. Reproductive age-associated fibrosis in the stroma of the mammalian ovary. Reproduction 2016;152(3):245-60.

[35] Scalercio SR, et al. Trolox enhances follicular survival after ovarian tissue autograft in squirrel monkey (Saimiri collinsi). Reprod Fertil Dev 2015.

[36] Lattouf R, et al. Picrosirius red staining: a useful tool to appraise collagen networks in normal and pathological tissues. J Histochem Cytochem 2014;62(10):751-8.

[37] Vogel W. Discoidin domain receptors: structural relations and functional implications. FASEB J 1999;13(Suppl):S77-82.

[38] Vogel W, et al. The discoidin domain receptor tyrosine kinases are activated by collagen. Mol.Cell 1997;1(1):13-23.

[39] Matsumura H, et al. Transcriptome analysis reveals an unexpected role of a collagen tyrosine kinase receptor gene, Ddr2, as a regulator of ovarian function. Physiol Genomics 2009;39(2):120-9.

[40] Manley PW, et al. Structural resemblances and comparisons of the relative pharmacological properties of imatinib and nilotinib. Bioorg Med Chem 2010;18(19):6977-86.

[41] Day E, et al. Inhibition of collagen-induced discoidin domain receptor 1 and 2 ac tivation by imatinib, nilotinib and dasatinib. Eur J Pharmacol 2008;599(13):44-53.

[42] Watkins WJ, et al. Mutational screening of FOXO3A and FOXO1A in women with premature ovarian failure. Fertil Steril 2006;86(5):1518-21.

[43] Kuscu N, Celik-Ozenci C. FOXO1, FOXO3, AND FOXO4 are differently expressed during mouse oocyte maturation and preimplantation embryo development. Gene Expr Patterns 2015;18(1-2):16-20.

[44] Pisarska MD, et al. Expression of forkhead transcription factors in human granulosa cells. Fertil Steril 2009;91(4 Suppl):1392-4.

[45] Ezzati MM, et al. Regulation of FOXO3 subcellular localization by kit ligand in the neonatal mouse ovary. J Assist Reprod Genet 2015;32(12):1741-7.

[46] Norambuena-Soto I, et al. Transforming growth factor-beta and Forkhead box O transcription factors as cardiac fibroblast regulators. Biosci Trends 2017;11(2):154-62.

[47] Adachi M, et al. The forkhead transcription factor FoxO1 regulates proliferation and transdifferentiation of hepatic stellate cells. Gastroenterology 2007;132(4):1434-46.

[48] Makker A, Goel MM, Mahdi AA. PI3K/PTEN/Akt and TSC/mTOR signaling path ways, ovarian dysfunction, and infertility: an update. J Mol Endocrinol 2014;53(3):R103-18.

[49] Alikhani M, Alikhani Z, Graves DT. FOXO1 functions as a master switch that regulates gene expression necessary for tumor necrosis factor-induced fibroblast apoptosis. J Biol Chem 2005;280(13):12096-102.

[50] Wang XW, Yu Y, Gu L. Dehydroabietic acid reverses TNF-alpha-induced the activation of FOXO1 and suppression of TGF-beta1/Smad signaling in human adult dermal fibroblasts. Int J Clin Exp Pathol 2014;7(12):8616-26.

[51] Lin $\mathrm{CH}$, et al. Resveratrol enhanced FOXO3 phosphorylation via synergetic activation of SIRT1 and PI3K/Akt signaling to improve the effects of exercise in elderly rat hearts. Age (Dordr) 2014;36(5):9705

[52] Pramod S, Shivakumar K. Mechanisms in cardiac fibroblast growth: an obligate role for Skp2 and FOXO3a in ERK1/2 MAPK-dependent regulation of p27kip1. Am J Physiol Heart Circ Physiol 2014;306(6):H844-55.

[53] Pei XM, et al. Protective effects of desacyl ghrelin on diabetic cardiomyopathy. Acta Diabetol 2015;52(2):293-306.

[54] Naka K, et al. TGF-beta-FOXO signalling maintains leukaemia-initiating cells in chronic myeloid leukaemia. Nature 2010;463(7281):676-80.

[55] Liang R, et al. Evidence for AKT-independent regulation of FOXO1 and FOXO3 in haematopoietic stem and progenitor cells. Cell Cycle 2016;15(6):861-7.

[56] Vittal R, et al. Effects of the protein kinase inhibitor, imatinib mesylate, on epithelial/mesenchymal phenotypes: implications for treatment of fibrotic diseases. Pharmacol Exp Ther 2007;321(1):35-44.

[57] Raimondi C, et al. Imatinib inhibits VEGF-independent angiogenesis by targeting neuropilin 1-dependent ABL1 activation in endothelial cells. J Exp Med 2014;211(6):1167-83.

[58] Luo J, et al. Vascular endothelial growth factor promotes the activation of hepati stellate cells in chronic schistosomiasis. Immunol Cell Biol 2017;95(4):399-407.

[59] Hinz B. Myofibroblasts. Exp Eye Res 2016;142:56-70.

[60] Challa AA, Stefanovic B. A novel role of vimentin filaments: binding and stabilization of collagen mRNAs. Mol Cell Biol 2011;31(18):3773-89.

[61] Kidd ME, Shumaker DK, Ridge KM. The role of vimentin intermediate filaments in the progression of lung cancer. Am J Respir Cell Mol Biol 2014;50(1):1-6.

[62] Sancho M, et al. Proliferation of interstitial cells in the cyclophosphamide-induced cystitis and the preventive effect of imatinib. Biomed Res Int 2017:2017:3457093.

[63] Nurmio M, et al. Adult reproductive functions after early postnatal inhibition by imatinib of the two receptor tyrosine kinases, c-kit and PDGFR, in the rat testis. Reprod Toxicol 2008;25(4):442-6. 